\title{
Quantum Transport in Graphene Nanoribbons with Realistic Edges
}

\author{
Patrick Hawkins, Milan Begliarbekov, Marko Zivkovic, Stefan Strauf, Christopher P. Search \\ Department of Physics and Engineering Physics, \\ Stevens Institute of Technology, Hoboken NJ 07030, USA
}

\begin{abstract}
Due to their unique electrical properties, graphene nanoribbons (GNRs) show great promise as the building blocks of novel electronic devices. However, these properties are strongly dependent on the geometry of the edges of the graphene devices. Thus far only zigzag and armchair edges have been extensively studied. However, several other self passivating edge reconstructions are possible, and were experimentally observed. Here we utilize the Nonequilibrium Green's Function (NEGF) technique in conjunction with tight binding methods to model quantum transport through armchair, zigzag, and several other self-passivated edge reconstructions. In addition we consider the experimentally relevant cases of mixed edges, where random combinations of possible terminations exist on a given GNR boundary. We find that transport through GNR's with self-passivating edge reconstructions is governed by the sublattice structure of the edges, in a manner similar to their parent zigzag or armchair configurations. Furthermore, we find that the reconstructed armchair GNR's have a larger band gap energy than pristine armchair edges and are more robust against edge disorder. These results offer novel insights into the transport in GNRs with realistic edges and are thus of paramount importance in the development of GNR based devices.
\end{abstract}

\section{INTRODUCTION}

The study of quantum transport in graphene nanoribbons (GNR's) is of particular interest for the development of novel nanoelectronic devices since, unlike in conventional materials, the transport characteristics of nanostructured graphene based devices are affected by the geometry (chirality) of the GNR edges. In the ideal case the edges of GNR's can be arranged in two distinct geometries: zigzag and armchair, as shown in Fig. 1A. Pristine armchair edges, i.e., armchair edges with no atomic roughness, are semiconducting [1, 2, while pristine zigzag edges are metallic [1, 2, as shown in Figs. $1 \mathrm{~B}$ and $1 \mathrm{C}$. , in which the current density through zigzag and armchair terminated GNR's is plotted, as calculated using the methodology described below. The homogenous current density in the zigzag GNR is a signature of the metallic density of states at the edges. Conversely, the current density of the armchair terminated GNR is inhomogeneous and shows several distinct paths of electronic carriers, known as quantum billiards [3, 4, which result from the reflection of carriers off of the semiconducting edges. The semiconducting nature of the armchair edge originates from a quantum confinement effect, whereas the metallic nature of the zigzag edge stems from a nondispersive state localized at the periphery of the GNR [5, 6]. The localized states stems from the fact that the bottom of the conduction band and the top of the valence band are always degenerate at the $k=\pi$ point in the Brilluoin zone. The origins of this degeneracy are discussed in detail in Ref. [2].

The contrasting behavior of chiral zigzag and armchair edges allows the possibility of utilizing armchair and zigzag GNR's for different electronic and photonic

*Electronic address: mbegliar@stevens.edu

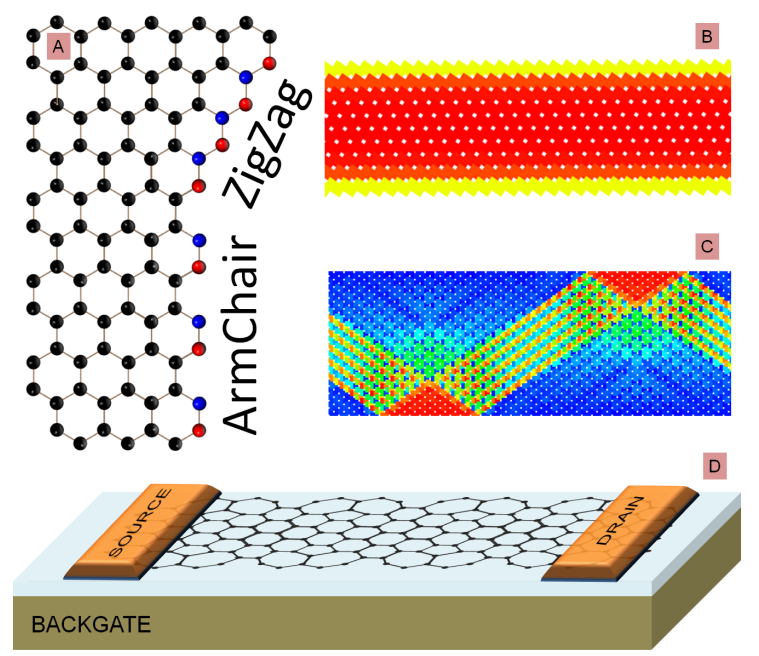

Figure 1: A A schematic of a graphene lattice highlighting the ideal armchair and zigzag edges. The edge atoms are colored red and blue to reflect the valleys to which they belong. Contour plots of the $2 \mathrm{D}$ current density through perfect $\mathbf{B}$ zigzag and $\mathbf{C}$ armchair nanoribbons, for the device geometry shown in panel $\mathbf{D}$. The reddish (bluish) colors correspond to high (low) current densities. Both ribbons are $10 \mathrm{~nm} \times 30$ $\mathrm{nm}$, backgate $V_{b g}$ bias is $0.01 \mathrm{eV}$, and an infinitesimal source drain bias.

applications. For example, armchair GNR's are ideal for use in high speed transistor [7, 8] and photodetector [9] devices that require a bandgap. The localized state of the zigzag GNR's is ferromagnetic [10] and is thus capable of rendering the GNR half-metallic under the application of a lateral electric field [11. Consequently, zigzag GNR's are ideal for spintronic applications. Furthermore combining zigzag and armchair edges in a single device results in novel behaviors such as current rectification in Z-shaped GNR's [12, 13] and spin filtering [14].

Although extensive theoretical models have been put 
forth to study transport through pristine armchair and zigzag GNR's, 15] little is known about transport through GNR's with realistic edges, i.e., edges that are not atomically pristine. Since all experimentally realized GNR's have thus far possessed some degree of edge roughness [16, 17, an understanding of transport through these devices is vital for the design of novel electronic devices. Furthermore, while ideal armchair and zigzag edges are the most frequently studied edge types, other self-passivating edge reconstructions are possible [18] and have been experimentally observed [19, 20]. The influence of partially and fully reconstructed edges on transport in GNR's has not yet been analyzed in detail. In this work we utilize nonequilibrium Green's functions (NEGF) methods to numerically simulate quantum transport through GNR's with both atomically smooth as well as rough edges. We furthermore simulate transport in self-passivated GNR's with various degrees of edge roughness. We find that transport through GNR's with self-passivating edge reconstructions is governed by the sublattice structure of the edges, in a manner similar to their parent zigzag or armchair configurations. Furthermore, we find that the reconstructed armchair GNR's have a larger band gap energy than pristine armchair edges and are more robust against edge disorder. Taken together our results elucidate the effects of edge reconstructions on the transport properties of GNR's and are thus of paramount importance for the design of novel electronic and photonic devices.

\section{A. Model}

Numerical simulations have been carried out with the aid of nonequilibrium Green's functions (NEGF) in conjunction with the tight binding formalism, where only the first order (nearest neighbor) hopping is considered. The simulations were implemented using the KNIT algorithm 21] on a 46 node parallel cluster. The system Hamiltonian is of the form

$$
\hat{H}=\sum_{i \neq j} \gamma_{i j} c_{i}^{\dagger} c_{j}+\sum_{i=1} \epsilon_{i} c_{i}^{\dagger} c_{j}
$$

where $\gamma_{i j}$ is the nearest neighbor hopping integral, $\epsilon_{i}$ is the on-site energy, and $c_{i}^{\dagger} / c_{j}$ are the creation / annihilation operators for the $i$-th and $j$-th lattice sites respectively. With the aid of the above Hamiltonian the local current $I_{i j}$ and density of states $\rho_{i}$ can be computed using the standard NEGF formalism according to

$$
\begin{gathered}
I_{i j}=\int d E\left[\gamma_{i j} G_{j i}^{<}(E)-\gamma_{i j} G_{i j}^{<}(E)\right] \text { and } \\
\rho_{i}=\frac{1}{2 \pi} \Im\left\{\int d E G_{i i}^{<}(E)\right\},
\end{gathered}
$$

where $G_{i j}^{<}(E)$ is the retarded nonequilibrium Green's function given by

$$
G_{i j}^{<}(E)=i \int d t e^{-i E t}\left\langle c_{j}^{\dagger} c_{i}(t)\right\rangle .
$$

The systems considered in this study consist of narrow graphene nanoribbons, typically $\sim 10$ x $30 \mathrm{~nm}$ connected to source-drain electrodes via Ohmic contacts. The Fermi energy $E_{F}$ may be adjusted via the application of a backgate bias 4] as shown in Fig. 1d. The above formalism is sufficient to describe transport through ideal armchair and zigzag GNR's. However, in order to properly account for the altered bond lengths of the self-passivated edge reconstructions [18], the environmentally dependent tight binding model was utilized [22, in which the change in the bond lengths is accounted for by modifying the values of the hopping integrals $\gamma_{i}$ according to

$$
\gamma_{i}\left(R_{j k}\right)=\alpha_{1} R_{j k}^{-\alpha_{2}} \exp \left(-\alpha_{3} R_{j k}^{-\alpha_{4}}\right)\left[1-S_{j k}\right]
$$

where $\alpha_{i}$ are the scaling parameters and $S_{j k}$ are the screening functions calculated from first principles calculations in Ref. [22]. $R_{j k}$ are the bond lengths of the edge reconstructions, which are calculated in Ref. [18. Following the results of Ref. [22], the screening function is of the form

$$
S_{k j}=\frac{\exp \left(\xi_{j k}\right)-\exp \left(-\xi_{j k}\right)}{\exp \left(\xi_{j k}\right)+\exp \left(-\xi_{j k}\right)}
$$

with

$$
\xi_{j k}=\beta_{1} \sum_{l} \exp \left[-\beta_{2}\left(\frac{R_{j l}+R_{k l}}{R_{j l}}\right)^{\beta_{3}}\right]
$$

where $\beta_{i}$ are the screening, and $R_{j l}$ and $R_{k l}$ are the nextnearest neighbor bond lengths. The bond lengths and the values of the nearest neighbor hopping integrals are listed in Fig. 2f.

Before proceeding to the discussion of our results, it should be noted that spin effects were previously predicted to play an important role [23, 24] in transport of pristine zigzag GNRs whose edge states result in flat bands at the Fermi energy. Spin effects may be included by adding a Hubbard term $H^{\prime}=U \sum_{i} n_{i \uparrow} n_{i \downarrow}$, to the Hamiltonian where $U$ is a parameter that defines the strength of the on-site Coulomb interaction. Previous calculations with zigzag GNRs have shown that $H^{\prime}$ leads to the development of ferromagnetic spin orientations along each edge and antiferromagnetic correlations between the two edges. The spin polarization of the edge states breaks the degeneracy of the flat energy bands at the Fermi energy with a gap opening up at $E_{F}$. This band gap leads to a vanishing of the density of 

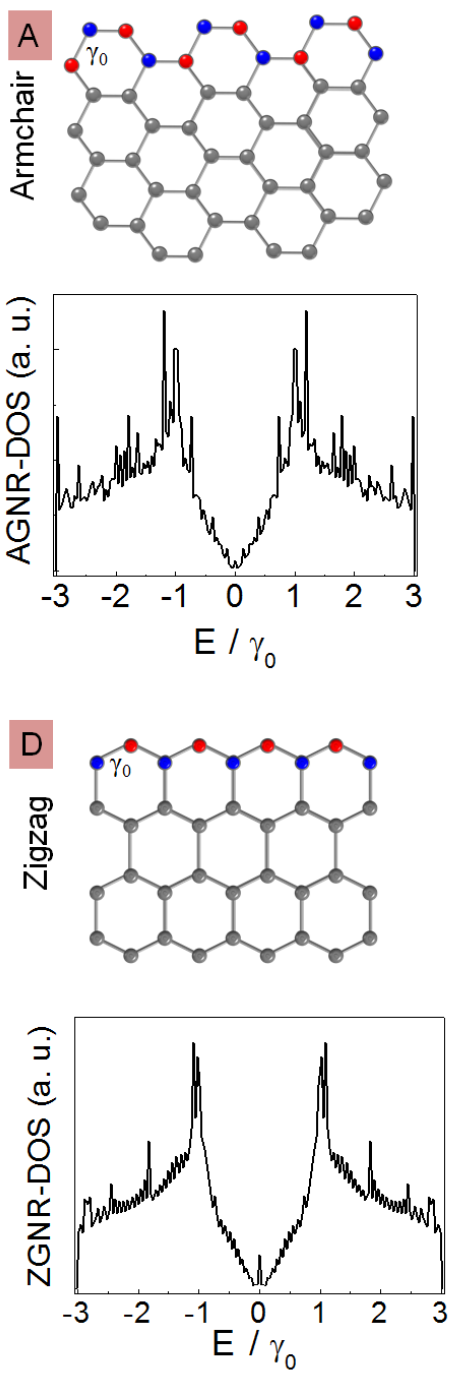
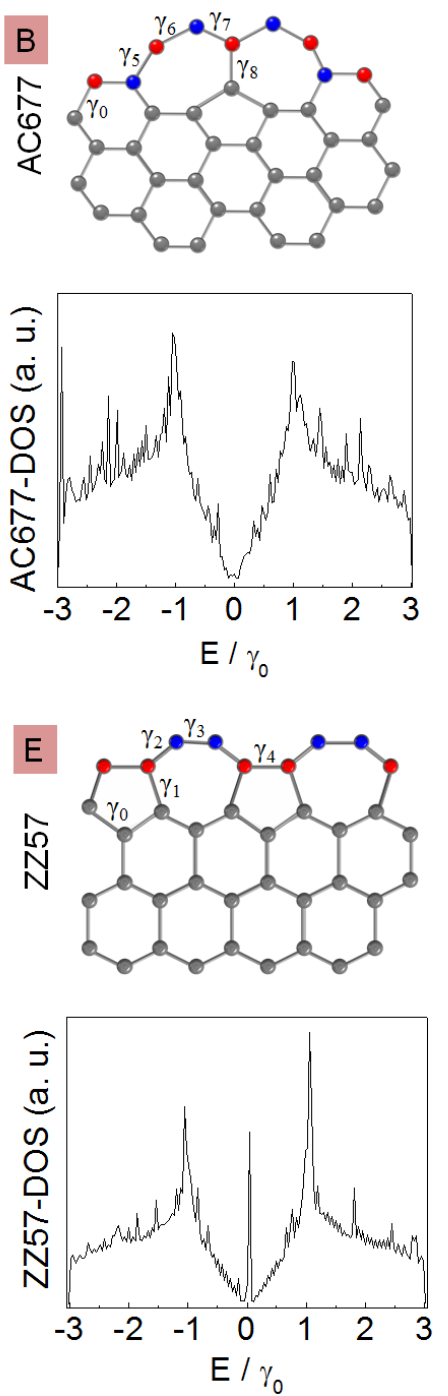
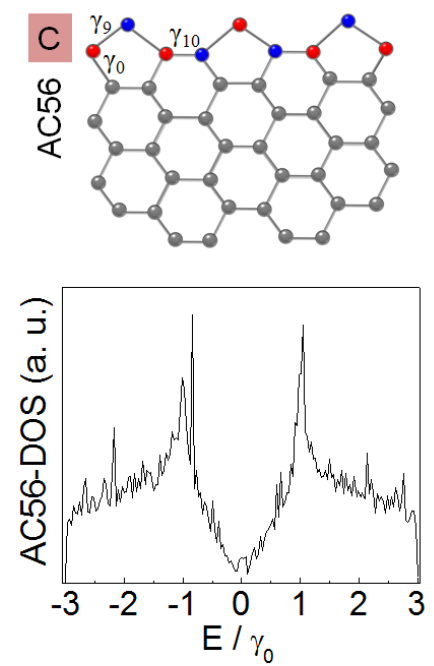

\begin{tabular}{|c|c|c|}
\hline$F$ & $\begin{array}{c}\text { Bond } \\
\text { Length }^{*}(\AA)\end{array}$ & $\begin{array}{c}\text { Hopping energy - } \\
\gamma_{\mathrm{i}} / \gamma_{0}\end{array}$ \\
\hline$\gamma_{0}$ & 1.42 & 1.0 \\
\hline$\gamma_{1}$ & 1.47 & 0.957 \\
\hline$\gamma_{2}$ & 1.41 & 1.009 \\
\hline$\gamma_{3}$ & 1.24 & 1.189 \\
\hline$\gamma_{4}$ & 1.44 & 0.982 \\
\hline$\gamma_{5}$ & 1.44 & 0.982 \\
\hline$\gamma_{6}$ & 1.24 & 1.189 \\
\hline$\gamma_{7}$ & 1.43 & 0.991 \\
\hline$\gamma_{8}$ & 1.4 & 1.018 \\
\hline$\gamma_{9}$ & 1.52 & 0.917 \\
\hline$\gamma_{10}$ & 1.63 & 0.838 \\
\hline \multicolumn{3}{|c|}{} \\
\hline
\end{tabular}

Figure 2: Illustrations of the edge reconstructions and their densities of states relative to the Fermi energy for the A armchair, B AC677, C AC56 D zigzag, and E ZZ57 nanoribbons. The table in F shows the values of the bond lengths (adopted from Ref. 22) and change in the hopping energy $\gamma_{i} / \gamma_{0}$, where the values of $\gamma_{i}(i=1,2 \cdots 10)$ are indicated in the diagrams in panels a-e. In these simulations we used $\gamma_{0}=2.8 \mathrm{eV}$.

states at $E_{F}$ while higher energy states are unaffected by $H^{\prime}[23,25,26]$. There is some experimental evidence for the energy splitting of the edge states [27+29]although there is a debate as to whether such magnetism could exist in real world conditions [26. Among the many effects that would quench this edge state magnetism is edge reconstruction. Edge reconstructed zigzag GNRs are expected to show no magnetic effects since the edge state energy bands at $E_{F}$ are no longer flat [25, 26]. Here we do not consider nonlinear spin effects in our results.

\section{RESULTS AND DISCUSSION}

Figure 2 shows the atomic arrangements of the five types of edges considered in this work: the armchair and zigzag edges as well as the three types of selfpassivating reconstructions, and their corresponding densities of states. Although pristine armchair and zigzag edges are expected for the honeycomb lattice geometry of graphene, thermal [30] and optical [17] edge reconstruction has been observed to give rise to the self passivating variants of the pristine edges. The most common mechanism of bond rearrangement in sp2 crystalline carbons is the Stone-Wales mechanism, which in the case of GNR edges can lead to the ZZ57 reconstruction. This mechanism has been studied extensively in relation to nanocrystalline carbon systems 31 33. Furthermore, the energetics of other self-passivating edge reconstructions were also investigated [18]. The names of the reconstructed edges originate from the parent edge type, i.e., zigzag or armchair, and the geometry of the closed structure. For 

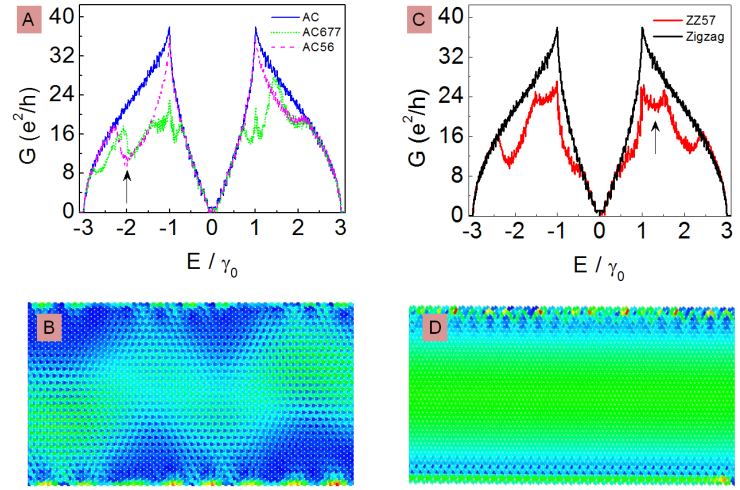

Figure 3: A Conductance of ideal armchair (solid blue), AC677 (dash-dotted green), and AC56 (dashed pink) lines. C Conductance through ideal zigzag (solid black) and ZZ57 (dotted red) lines. The 2D current density for B AC56 and D ZZ57 reconstructions for the same parameters as in Fig. 1.

example, the ZZ57 edge is a zigzag edge that is terminated by alternating pentagons and heptagons. As can be seen in Fig. 2, the self passivating edge reconstructions together with the unaltered edges form two distinct families of edges: the zigzag and the armchair family. Although the reconstructed edges bear little resemblance to their parent edge types, the zigzag reconstruction is similar to the pristine zigzag edge in that it possesses a metallic state at $E=0$, as can be seen in Figs. 2D and $2 \mathrm{E}$. Interestingly, the magnitude of the $E=0$ state is found to be larger for the ZZ57 reconstruction than for the zigzag GNR of the same width. Consequently, ZZ57 edges might be of greater utility for devises that exploit the edge localized states, such as devices based on graphene antidot superlattices [6]. Phenomenologically, the increase in the magnitude of the $E=0$ state can be attributed to Anderson localization that arises from the additional break in the periodicity of the hexagonal lattice at the ZZ57 edge, as can be seen in Figs. 2D and 2E. Similarly both of the armchair reconstructions are semiconducting and possess a finite energy gap as discussed in detail below. The similarities between the parent edge types and their reconstructed counterparts can be readily understood by considering the sublattice structure of the terminating atoms at the edges. Both zigzag and ZZ57 edges are terminated by atoms that belong to the same sublattice, whereas armchair as well as AC677 and AC56 edges contain atoms that belong to both sublattices. The terminating atoms are atoms that are the outermost atoms of the GNR. Consequently, the armchair edges support intervalley scattering, whereas the zigzag edges do not. Thus the sublattice structure of the terminating atoms in the self passivating edge reconstructions determines the properties of the resultant edges in a manner identical to the pristine armchair and zigzag edges.

Having discussed the principle features of the zigzag and armchair families we now turn to the discussion of transport through GNR's with self passivating edge reconstructions. We first restrict the discussion to fully reconstructed edges. Figures $3 \mathrm{~A}$ and $3 \mathrm{C}$ show the conductance of pristine and reconstructed armchair and zigzag terminated GNR's respectively. Figures 3B and 3D show the 2-D current density of AC56 and ZZ57 edges. Furthermore the 2-D current density for the AC677 reconstruction was found to be similar to that of the AC56 edge (data not shown). The prominent features in the conductance through GNRs with reconstructed edges are the dips in the conductance at certain values of the Fermi energy. These dips arise from the fact that the size of the unit cell is enlarged at the reconstructed edges. Consequently, fewer states are available per unit area as compared to the unreconstructed edges resulting in pronounced dips in the conductance. It should be further noted that these dips appear energetically far away from the Dirac point. These features can potentially be probed in either ARPES or optical conductivity experiments. However, since no dips are observed in the vicinity of the Dirac point, the low bias conductance of reconstructed GNR's should be similar to the conductance of pristine GNR's. Therefore, our results demonstrate that while transport in narrow GNR's is highly sensitive to the type of edge terminations, namely, zigzag or armchair, it is robust with regard to self-passivating edge reconstructions within the same edge family. Consequently, the relevant physical mechanisms that dominate transport in armchair and zigzag terminated GNR's can be still observed in GNR's with realistic edge reconstructions.

The 2D current density of AC56 and ZZ57 edges further highlights the similarity between the reconstructed and pristine GNR edges. The AC56 terminated GNR's show an oscillatory current density characteristic of quantum billiards in pristine armchair GNRs, whereas the current density in the ZZ57 terminated GNR is homogenous. The most striking difference in the $2 \mathrm{D}$ current density between pristine and reconstructed edges is the presence of edge-localized charge density. Phenomenologically this effect can be understood in terms of Anderson localization since the reconstructed edges break the periodicity of the underlying lattice thereby localizing the electronic wavefunctions. As a result the quantum billiard patterns are smeared by the edge-localized modes.

Having analyzed the effects of complete reconstruction on transport through GNR's we now turn our attention to the case of partial reconstructions. To study the effects of partial reconstructions on transport through GNR's we first calculate the conductance of a pristine armchair and zigzag GNR and then introduce various percentages of AC677 and ZZ57 edges respectively. To quantify the effect of reconstruction, we plot the conductance value of two dips $(E=2.1 \mathrm{eV}$ for the armchair reconstruction and $E=1.1 \mathrm{eV}$ for the zigzag reconstruction) as shown in Fig. 4. The dips are identified by black arrows in Figs. 3Aand 3C. As can be seen in Fig. 4 the onset of the dips occurs at small percentages of edge reconstructions $(\sim 5 \%)$. Increasing the amount of 


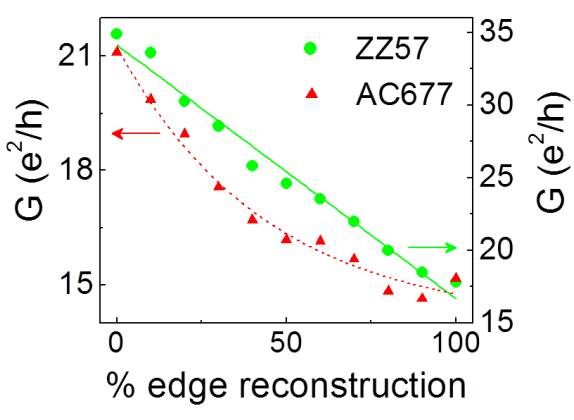

Figure 4: Conductance of an armchair (red triangles) and a zigzag (green circles) nanoribbons with varying percentages of edge reconstruction with AC677 and ZZ57 substitutions respectively. The conductance values were extracted for $E_{F}=$ 2.1 and $E_{F}=1.1 \mathrm{eV}$ for the armchair and zigzag nanoribbons respectively, as indicated by the arrows in Fig. 3a and 3c, for the same size devices as indicated in Fig. 1.

edge reconstruction increases the amplitude of the dips, which become most pronounced for fully reconstructed GNR's. It should be noted that the armchair reconstructions have a more dramatic effect on the conductivity than the zigzag reconstructions. The magnitude of the dip in the conductance of an armchair GNR reconstructed by AC677 segments decreases exponentially according to $G\left(\% x_{\mathrm{AC} 677}\right)=A_{1} \exp \left(-\% x_{\mathrm{AC} 667} / A_{2}\right)+A_{3}$, where $\% x_{\mathrm{AC} 667}$ is the percentage of AC677 segments and $A_{1}=8.1, A_{2}=44.1$, and $A_{3}=13.0$ are empirical fit parameters in units of $e^{2} / \mathrm{heV}$. The magnitude of the dip in the conductance of a zigzag GNR decreases linearly as a function of ZZ57 reconstruction according to $G\left(\% x_{\mathrm{ZZ} 57}\right)=B_{1}\left(\% x_{\mathrm{ZZ} 57}\right)+B_{2}$, where $\% x_{\mathrm{ZZ} 57}$ is the percentage of ZZ57 reconstruction and $B_{1}=-0.17$ and $B_{2}=34.2$ are empirical fit parameters in units of $e^{2} / \mathrm{heV}$.

Finally, we turn our attention to the bandgap of reconstructed armchair GNR's. In light of the above discussion it is sufficient to only consider fully reconstructed GNR's since only the absolute magnitude of the bandgap is effected by partially reconstructed GNR's. The gap energy, $E_{G}$, is extracted from the conductance of the GNR. Figures $5 \mathrm{a}$ and $5 \mathrm{~b}$ show the magnitude of $E_{G}$ as a function of ribbon width for AC677 and AC56 ribbons respectively. As can be seen in Fig. 4, the bandgap of very narrow GNR's less than $4 \mathrm{~nm}$ surpasses $1 \mathrm{eV}$; however, in both cases it decreases exponentially as a function of ribbon width. For the AC677 terminated ribbon the gap energy as a function of ribbon width $E_{G}^{(A C 667)}(n)$ decreases according to $E_{G}^{(A C 667)}(n)=C_{1} \exp \left(-n / C_{2}\right)+C_{3}$, where $n$ is the number of atoms that span the width $N$ of the GNR, and $C_{1}=2.1, C_{2}=17.5$, and $C_{3}=0.2$ are empirical fit parameters in units of $e^{2} / \mathrm{heV}$. Similarly, $E_{G}^{(A C 56)}(n)$ for the AC56 ribbons varies according to $E_{G}^{(A C 56)}(n)=D_{1} \exp \left(-n / D_{2}\right)+D_{3}$, where $D_{1}=1.7$, $D_{2}=17.8$, and $D_{3}=0.2$ are empirical fit parameters in units of $e^{2} / h e V$. A striking difference between the AC677
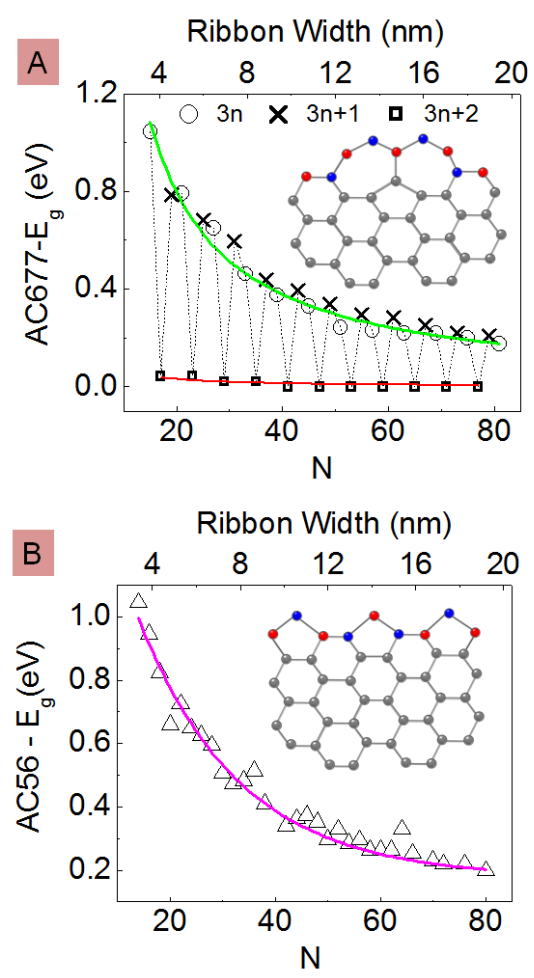

Figure 5: Bandgap as a function of ribbon width for A AC677 and B AC56 nanoribbons, for the same device parameters as indicated in Fig. 1. $n$ is the number of Carbon atoms that span the width $N$ of the GNR. The different symbols in panel 'a' represent the multiplicity of the number of atoms that span the width of the GNR. The circles, crosses, and squares indicate GNR's of widths that are multiples of $N=3 n, N=$ $3 n+1$, and $N=3 n+2$ respectively.

and AC56 GNR's is that the AC677 possess an additional family dependence of the gap energy on the multiplicity of the number of atoms that span the width GNR. Namely, AC677 terminated GNR's with width's that are multiples of $3 n$ and $3 n+1$ possess an electronic bandgap, whereas AC677 terminated GNR's with widths that are multiples of $3 n+2$ do not have a bandgap. The same family dependence on the width multiplicity was previously calculated for pristine armchair terminated GNR's 34 36. This behavior, however, vanishes in AC56 terminated GNR's. The lack of the multiplicity dependence of $E_{G}$ in AC56 terminated GNR's can be understood from the fact that the AC56 geometry imposes a greater strain on the underlying lattice, thereby distorting more of the features of pristine armchair GNR's. Finally, it is interesting to note that $E_{G}$ in both AC677 and AC56 terminated ribbons is twice as large as $E_{G}$ in pristine armchair nanoribbons of the same width (see, for example, the numerical calculations of the armchair bandgap in Ref. 34]). Consequently, these variants of the armchair GNR's might become more useful candidates for GNR based devices, if a reliable method for fabricating these edges is found. 


\section{SUMMARY}

In summary, we simulated quantum transport through graphene nanoribbons with realistic edge terminations by utilizing nonequilibrium Green's functions. We found that transport in the self-passivated edge reconstructions is qualitatively similar to transport in the parent edge type. This similarity is attributed to the sublattice structure of the terminating atoms at the edges. We also found that all of the self passivating reconstructions localize electron wavefunctions at the edge. This localization may be understood in terms of Anderson localization since the reconstructed edges further break the periodicity of the underlying lattice. We further analyzed the width dependence of the bandgap in the reconstructed armchair GNR's, and found that the gap energy is twice as large as the gap energy of pristine armchair GNR's of the same width. Finally, we have shown that the band gap in AC56 terminated GNR's is more robust against the multiplicity, which is of advantage for fabrication which inevitably introduces width fluctuations. Taken together, these results elucidate the nature of quantum transport in graphene based devices with realistic edges and are thus important for the development of future graphene based nanoelectronic devices.
[1] Malard, L.; Pimenta, M.; Dresselhaus, G.; Dresselhaus, M. Physics Reports 2009, 473, 51.

[2] Nakada, K.; Fujita, M.; Dresselhaus, G.; Dresselhaus, M. Phys. Rev. B 1996, 54, 1795.

[3] Ponomarenko, L. A.; Schedin, F.; Katsnelson, M. I.; Yang, R.; Hill, E. W.; Novoselov, K. S.; Geim, A. K. Science 2008, 320, 356-358.

[4] Miao, F.; Wijeratne, S.; Zhang, Y.; Coskun, U. C.; Bao, W.; Lau, C. N. Science 2007, 317, 1530-1533.

[5] Wimmer, M.; Akhmerov, A. R.; Guinea, F. Physical Review B 2010, 82, 045409.

[6] Begliarbekov, M.; Sul, O.; Santanello, J.; Ai, N.; Zhang, X.; Yang, E.-H.; Strauf, S. Nano Letters 2011, 11, 1254-1258.

[7] Lin, Y. . M.; Dimitrakopoulos, C.; Jenkins, K. A.; Farmer, D. B.; Chiu, H. . Y.; Grill, A.; Avouris, P. Science 2010, 327, 662-662.

[8] Begliarbekov, M.; Strauf, S.; Search, C. P. Nanotechnology 2011, 22, 165203.

[9] Xia, F.; Mueller, T.; Lin, Y.-m.; Valdes-Garcia, A.; Avouris, P. Nature Nanotechnology 2009, 4, 839-843.

[10] Jiang, D.-E.; Sumpter, B. G.; Dai, S. Journal of Chemical Physics 2007, 127, 124703.

[11] Son, Y.-W.; Cohen, M. L.; Louie, S. G. Nature 2006, 444, 347-349.

[12] Wang, Z. F.; Shi, Q. W.; Li, Q.; Wang, X.; Hou, J. G.; Zheng, H.; Yao, Y.; Chen, J. Applied Physics Letters 2007, 91, 053109.

[13] Wang, Z. F.; Li, Q.; Shi, Q. W.; Wang, X.; Hou, J. G.; Zheng, H.; Chen, J. Applied Physics Letters 2008, 92, 133119.

[14] Saffarzadeh, A.; Farghadan, R. Applied Physics Letters 2011, 98, 023106.

[15] Wakabayashi, K.; Fujita, M.; Ajiki, H.; Sigrist, M. Physical Review B 1999, 59, 8271-8282.

[16] Begliarbekov, M.; Sul, O.; Kalliakos, S.; Yang, E.-H.; Strauf, S. Applied Physics Letters 2010, 97, 031908.

[17] Begliarbekov, M.; Sasaki, K.-I.; Sul, O.; Yang, E.-H.; Strauf, S. Nano Letters 2011, 11, 4874-4878.

[18] Koskinen, P.; Malola, S.; Hakkinen, H. Physical Review Letters 2008, 101, 115502.

[19] Koskinen, P.; Malola, S.; Haekkinen, H. Physical Review $B$ 2009, 80, 073401.
[20] Girit, C. O.; Meyer, J. C.; Erni, R.; Rossell, M. D.; Kisielowski, C.; Yang, L.; Park, C.-H.; Crommie, M. F.; Cohen, M. L.; Louie, S. G.; Zettl, A. Science 2009, 323, 1705-1708.

[21] Kazymyrenko, K.; Waintal, X. Physical Review B 2008, 77, 115119.

[22] Tang, M. S.; Wang, C. Z.; Chan, C. T.; Ho, K. M. Physical Review B 1996, 53, 979-982.

[23] Yazuev, O. V. Rep. Prog. Phys. 2010, 73, 056501.

[24] Girao, E. C.; Liang, L.; Cruz-Silva, E.; Filho, A. G. S.; Meunier, V. Phys. Rev. Lett. 2011, 10\%, 135501.

[25] Song, L.; Zheng, X. H.; Wang, R. L.; Zeng, Z. J. Phys. Chem. C 2010, 114, 12145-12150.

[26] Kunstmann, J.; Ozdogan, C.; Quandt, A.; Fehske, H. Physical Review B 2011, 83, 045414.

[27] Pan, M.; Costa Girao, E.; Jia, X.; Bhaviripudi, S.; Li, Q.; Kong, J.; Meunier, V.; Dresselhaus, M. S. Nano Letters 2012, 12, 1928-1933.

[28] Rao, S. S.; Jammalamadaka, S. N.; Stesmans, A.; Moshcalkov, V. V.; van Tol, J.; Kosynkin, D. V.; Higginbotham-Duque, A.; Tour, J. M. Nano Letters 2012, 12, 1210-1217.

[29] Tao, C.; Jiao, L.; Yazyev, O. V.; Chen, Y.-C.; Feng, J.; Zhang, X.; Capaz, R. B.; Tour, J. M.; Zettl, A.; Louie, S. G.; Dai, H.; Crommie, M. F. Nature Physics 2011, 7, 616-620.

[30] Xu, Y. N.; Zhan, D.; Liu, L.; Suo, H.; Ni, Z. H.; Nguyen, T. T.; Zhao, C.; Shen, Z. X. Acs Nano 2011, 5, 147-152.

[31] Ertekin, E.; Chrzan, D. C.; Daw, M. S. Physical Review B 2009, 79, 155421.

[32] Ma, J.; Alfe, D.; Michaelides, A.; Wang, E. Physical Review B 2009, 80, 033407.

[33] Fan, B. B.; Yang, X. B.; Zhang, R. Physics Letters A 2010, 374, 2781-2784.

[34] Yang, L.; Park, C.-H.; Son, Y.-W.; Cohen, M. L.; Louie, S. G. Physical Review Letters 2007, 99, 186801.

[35] Wakabayashi, K.; Sasaki, K.-i.; Nakanishi, T.; Enoki, T. Science and Technology of Advanced Materials 2010, 11, 054504.

[36] Zheng, H.; Wang, Z. F.; Luo, T.; Shi, Q. W.; Chen, J. Physical Review B 2007, 75, 165414. 